\title{
ASSESSING STATUS AND HABITAT OF SIAMESE FIREBACK (Lophura diardi) BY USING CAMERA TRAP IN LO GO-XA MAT NATIONAL PARK, VIETNAM
}

\author{
Nguyen Tran Vy1,*, To Quang², Ho Dac Long2, Hoang Van Hai ${ }^{2}$, \\ Huynh Huu Phuong ${ }^{2}$, Nguyen Long Dien ${ }^{2}$, Nguyen Minh Tan ${ }^{3}$ \\ ${ }^{1}$ Institute of Tropical Biology, VAST, Vietnam \\ ${ }^{2}$ Lo Go-Xa Mat National Park, Tay Ninh Province, Vietnam \\ ${ }^{3}$ Department of Science and Technology, Tay Ninh Province, Vietnam
}

Received 17 October 2019, accepted 10 February 2020

\begin{abstract}
Siamese fireback, Lophura diardi, belonging to Galliformes is at risk due to habitat loss and hunting, which are main challenges to conservation of the species. In order to provide scientific information to conserve the L. diardi, we implemented a camera-trap survey in Lo Go-Xa Mat National Park from 2017 to 2018. Occupancy and Poisson regression models were used to investigate presence of the species and potential factors influencing the occurrence of the species in the study area. Our results showed that the probability of occurrence of the species at locations within the park was high at $0.84(0.69-0.92)$ and detection probability was relative low at 0.19 $(0.16-0.23)$. The best models consistently suggested that the abundance of the species was high in closed canopy forest cover $(\beta=0.41)$, but appeared to be notably lower in areas far away from the ranger stations $(\beta=-0.25)$ and in places with high frequency of human $(\beta=-0.22)$. This research provided the first quantitative information of status and potential factors influencing occurrence of the L. diardi in the park, which is an essential data for developing practical actions to protect the species and monitoring program in the future for the park.
\end{abstract}

Keywords: Lophura diardi, species conservation, camera trap, Siamese fireback, Lo Go-Xa Mat.

Citation: Nguyen Tran Vy, To Quang, Ho Dac Long, Hoang Van Hai, Huynh Huu Phuong, Nguyen Long Dien, Nguyen Minh Tan, 2020. Assessing status and habitat of siamese fireback (Lophura diardi) by using cameratrap in Lo Go-Xa Mat National Park, Vietnam. Tap chi Sinh hoc, 42(1): 51-60. https://doi.org/10.15625/0866$7160 / \mathrm{v} 42 \mathrm{n} 1.14504$.

*Corresponding author email: vychim@yahoo.com

(2020 Vietnam Academy of Science and Technology (VAST) 


\title{
SỬ DỤNG BÃ̃Y CHỤP ẢNH ĐÁNH GIÁ HIỆN TRẠNG VÀ SINH CẢNH SỐNG CỦA GÀ LỒI HÔNG TÍA (Lophura diardi) Ở VƯờn QUỐC GIA LÒ GÒ-XA MÁT, VIỆT NAM
}

\author{
Nguyễn Trần V ỹ 1,**, Tô Quang², Hồ Đắc Long², Hoàng Văn Hải2, \\ Huỳnh Hữu Phương ${ }^{2}$, Nguyê̂n Long Điền ${ }^{2}$, Nguyễn Minh Tần ${ }^{3}$ \\ ${ }^{1}$ Viện Sinh học Nhiệt đới, Viện Hàn lâm Khoa học và Công nghệ Việt Nam \\ ${ }^{2}$ Vườn Quốc gia Lò Gò-Xa Mát, Tây Ninh \\ ${ }^{3}$ Sở Khoa học và Công nghệ Tây Ninh
}

Ngày nhận bài 17-10-2019, ngày chấp nhận 10-2-2020

\section{TÓM TẮT}

Gà lôi hông tía, Lophura diardi, thuộc Bộ gà (Galliformes) đang bị đe dọa do mất nơi sinh sống và săn bắt. Đây là những thách thức lớn đối với bảo tồn loài. Nhằm cung cấp cơ sở khoa học cho bảo tồn loài gà lôi hông tía, chúng tôi đã sử dụng bẫy chụp ảnh để nghiên cứu tại Vườn quốc gia Lò Gò-Xa Mát từ năm 2017 đến năm 2018. Mô hình xác suất hiện diện loài và hàm hồi quy phân phối Poisson được sử dụng để đánh giá sự có mặt và xác định các yếu tố có thể ảnh hưởng đến sự hiện diện của loài trong khu vực nghiên cứu. Kết quả nghiên cứu cho thấy xác suất hiện diện của loài cao ở mức $0,84(0,69-0,92)$ và xác suất bắt gặp loài tương đối thấp ở mức $0,19(0,16-0,23)$. Những mô hình chỉ ra độ phong phú của loài cao trong sinh cảnh rừng có độ che phủ cao $(\beta=0,41)$, nhưng thấp ở những khu vực xa trạm bảo vệ rừng $(\beta=$ $0,25)$ và ở những nơi có tần suất hoạt động của con người cao $(\beta=-0,22)$. Đây là dữ liệu định lượng đầu tiên về hiện trạng và các yếu tố ảnh hưởng đến sự hiện diện của $L$. diardi ở Vườn quốc gia Lò Gò-Xa Mát làm cơ sở khoa học giúp chương trình giám sát và bảo tồn loài Gà lôi hông tía, L. diardi, ở đây.

Từ khóa: Lophura diardi, bảo tồn loài, bẫy chụp ảnh, gà lôi hông tía, Lò Gò-Xa Mát.

*Địa chỉ liên hệ email: vychim@yahoo.com

\section{MỞ ĐẦU}

Trên thế gới, trong tổng số 308 loài thuộc bộ Gà (Galliformes), có khoảng 25\% thuộc nhóm các loài có nguy cơ bị tuyệt chủng cao (Hilton-Taylor et al., 2009; IUCN, 2019). Việt Nam có 22 loài thuộc họ chim Trĩ (Phasianidae) trong đó có một loài rất nguy cấp $(\mathrm{CR})$, một loài nguy cấp $(\mathrm{EN})$, bốn loài sắp nguy cấp (IUCN, 2019). Hiện nay, ngoài sinh cảnh sống bị suy giảm (Vy et al., 2017a; Wege et al., 1999), săn bắt vẫn là mối đe dọa hàng đầu đối với đa dạng sinh học ở Việt Nam, đặc biệt là săn bắt bằng bẫy dây cáp (bẫy giò) (Gray et al., 2018). Hình thức săn bắt này có ảnh hưởng rất lớn đến các loài sống trên nền đất trong đó có các loài chim Trĩ mặc dù chúng không phải là mục tiêu săn bắt (McGowan \& Garson, 2002). Tuy là nhóm đối tượng đang bị tác động nhiều, những nghiên cứu về sinh thái học, hiện trạng bảo tồn cũng như phân bố của các loài chim Trĩ ở Đông Nam Á nói chung, Việt Nam nói riêng rất ít (Brickle et al., 2008; Grainger et al., 2018). Thiếu dữ liệu khoa học về hiện trạng và sinh thái của các loài dẫn đến những khó khăn trong việc xây dựng các kế hoạch quản lý bảo tồn nhằm hạn chế 
nguy cơ tuyệt chủng của chúng trong đó có các lòai chim trĩ (Duckworth et al., 2012; Grainger et al., 2017).

Vườn quốc gia Lò Gò-Xa Mát (VQGLGXM), có ít nhất 5 loài thuộc họ chim Trĩ trong đó có gà tiền mặt đỏ (Polyplectron germaini) (phân hạng bảo tồn sắp nguy cấp $(\mathrm{NT})$ ) và gà lôi hông tía $(L$. diardi) (phân hạng bảo tồn ít lo ngại LC) (BirdLife International, 2019). Phân hạng bảo tồn của gà tiền mặt đỏ và gà lôi hông tía trong sách đỏ Việt Nam là sẽ nguy cấp (VU) (Anonymous, 2007b). Tuy nhiên đến nay hầu như không còn ghi nhận gà tiền mặt đỏ (Anonymous, 2007a) và chưa có những dữ liệu về hiện trạng của gà lôi hông tía ở đây. Trong nghiên cứu này chúng tôi tập trung đánh giá: 1) xác suất hiện diện và xác suất bắt gặp gà lôi hông tía tại VQG-LGXM; 2) đánh giá một số yếu tố có thể ảnh hưởng đến sự hiện diện của loài tại VQG-LGXM; 3) xác định mối đe dọa đến sự hiện diện của gà lôi hông tía tại VQG-LGXM.

\section{VÂTT LIỆU VÀ PHƯƠNG PHÁP NGHIÊN CỨU}

\section{Địa điểm nghiên cứu}

VQG-LGXM thuộc huyện Tân Biên, tỉnh Tây Ninh $\left(191,97 \mathrm{~km}^{2}, 1^{\circ} 02^{\prime}-11^{\circ} 47^{\prime} \mathrm{N}\right.$, $105^{\circ} 57^{\prime}-106^{\circ} 04^{\prime} E$. Toàn VQG-LGXM có 17 trạm bảo vệ rừng được bố trí trên trục lộ chính bao quanh và một trạm Đa Ha nằm bên trong VQG-LGXM (hình 1). Địa hình khá bằng phẳng, độ cao dao động từ 5-25 m so với mực nước biển và độ dốc từ $1-5^{\circ}$. Thảm thực vật của VQG-LGXM bao gồm các sinh cảnh chính như rừng thường xanh $(76 \%)$, bán rụng lá xen cây bụi $(3 \%)$, rừng rụng lá ngập nước theo mùa $(2 \%)$, trảng cỏ $(4.3 \%)$ và một số sinh cảnh khác. Tại VQG-LGXM, mùa mưa kéo dài từ tháng 6 đến tháng 10 với lượng mưa trung bình năm khoảng $1.800 \mathrm{~mm}$, mùa khô kéo dài từ tháng 11 đến tháng 5 năm và nhiệt độ trung bình năm $26,9^{\circ} \mathrm{C}$ (Anonymou s, 2007a).

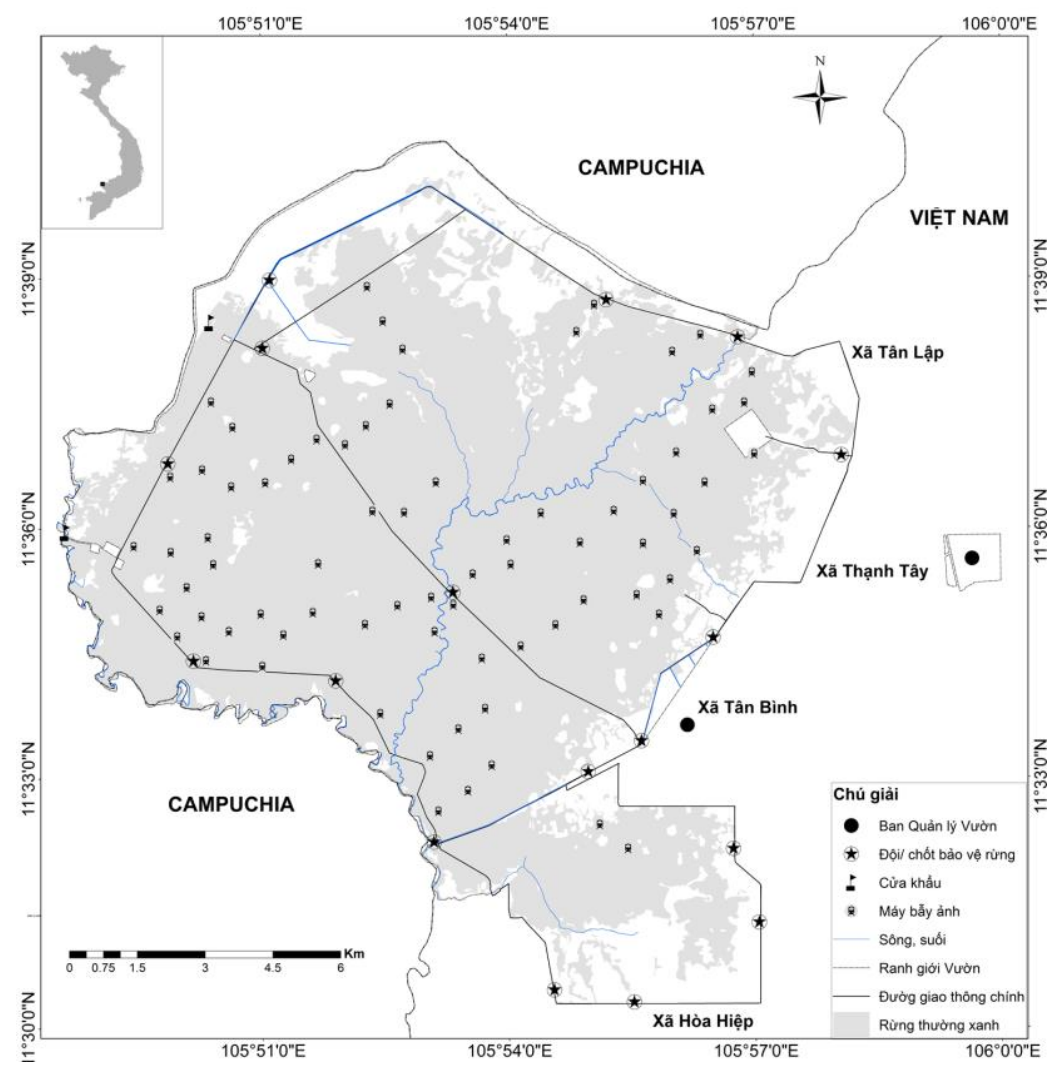

Hình 1. Vị trí hành chính của Vườn Quốc gia Lò Gò-Xa Mát và vị trí bẫy chụp ảnh 


\section{Đặt bẫy chụp ảnh}

Sử dụng 74 bẫy chụp ảnh (camera traps) cảm biến chuyển động và hồng ngoại (hiệu Bushnell Tropy Cam HD Brown, model 119874C) và đặt liên tục từ 2 đến 3 tháng trong sinh cảnh rừng thường xan $\mathrm{h}$ từ tháng $11 \mathrm{năm}$ 2017 đến tháng 5 năm 2018 để đảm bảo giả định một quần thể đóng về thời gian (MacKenzie, 2006). Vị trí các bẫy chụp ảnh được đặt cách xa nhau ít nhất là $700 \mathrm{~m}$ để thỏa mãn giả định tính độc lập của các ghi nhận giữa các máy ảnh (MacKenzie, 2006) (hình 1). Để ghi nhận hình ảnh của gà lôi hông tía tốt nhất, bẫy chụp ảnh được gắn trên gốc cây cách mặt đất từ $30-40 \mathrm{~cm}$ và được cài đặt chế độ chụp 3 hình liên tục cho mỗi lần chụp với khoảng thời gian giữa hai lần chụp là 0,3 giây.

\section{Điều tra số liệu về sinh cảnh sống}

Độ che phủ và độ dày của thảm thực vật rừng là những yếu tố môi trường tự nhiên có ảnh hưởng đến các loài thuộc họ chim Trĩ trong các nghiên cứu trước đây (Sukumal et al., 2015; Suwanrat et al., 2014; Vy et al., 2017a). Các yếu tố có liên quan đến con người như khoảng cách đến các trạm bảo vệ rừng, tần suất hiện diện của con người và những tác động của họ trong khu vực nghiên cứu được dùng để đánh giá hiệu quả công việc tuần tra của các trạm bảo vệ rừng (Ghoddousi et al., 2016; Hossain et al., 2016; Jenks et al., 2012). Vì vậy các yếu tố trên được thu thập để đánh giá sự ảnh hưởng của chúng lên phân bố của gà lôi hông tía tại VQG-LGXM. Dữ liệu độ che phủ của rừng và độ dày của thảm thực vật được thu thập theo tài liệu hướng dẫn (Abrams et al., 2018) và được xử lý bằng phần mềm GIMP phiên bản 2.10.12, R phiên bản 3.5.3 (R Development Core Team, 2019). Khoảng cách từ bẫy chụp ảnh đến các đội bảo vệ rừng được tính bằng phần mềm ArcGIS phiên bản 10.3 (ESRI, Redlands, USA). Tần suất hiện diện của con người là số lần xuất hiện của con người tại từng bẫy chụp ảnh.

Hình ảnh từ các bẫy chụp ảnh được kiểm tra xác định $L$. diardi và những người xuất hiện trong khu vực nghiên cứu. Sự xuất hiện và xác suất bắt gặp trung bình của $L$. diardi trong khu vực nghiên cứu được đánh giá bằng mô hình xác xuất hiện diện loài (không có các yếu tố môi trường) (MacKenzie, 2006). Để tăng độ chính xác của các mô hình, chúng tôi kết hợp dữ liệu của 6 ngày liên tục thành một lần khảo sát (Rovero et al., 2010; Tilker et al., 2018) để tạo thành 11 lần khảo sát lặp lại cho tất cả các bẫy chụp ảnh trong nghiên cứu này . Thời gian giữa các ghi nhận độc lập về $L$. diardi phải cách nhau ít nhất 60 phút kề từ lần ghi nhận sau cùng trước đó nhằm tránh trường hợp đếm lặp lại nhiều lần đối với những cá thể hiện diện quá lâu trước bẫy chụp ảnh (MacKenzie, 2006; Mugerwa et al., 2013). Tất cả các bước xử lý dữ liệu trên đều thực hiện bằng phần mềm $\mathrm{R}$ phiên bản $3.5 .3(\mathrm{R}$ Development Core Team, 2019) với các gói camtrapR phiên bản 0.99.9 (Niedballa et al., 2017) và gói Unmarked phiên bản $0.12-3$ (Fiske et al., 2019).

Các mô hình tương quan phân phối Poisson được sử dụng để đánh giá sự ảnh hưởng của các yếu tố môi trường lên sự hiện diện của $L$. diardi trong khu vực khảo sát. Số lượng $L$. diardi của mối lần khảo sát lặp lại trong mô hình là số lượng cá thể của lần ghi nhận cao nhất trong tất cả các lần ghi nhận độc lập của lần khảo sát lặp lại đó. Mô hình tương quan thích hợp nhất được lựa ch ọn dựa vào chỉ số AICc (Akaike Information Criterion) (Akaike, 1973) với chỉ số AICc thấp nhất và chỉ số wi (Akaike model weights) cao nhất (Burnham \& Anderson, 2002). Các biến độc lập liên tục được chuẩn hóa (Gelman, 2008) và kiểm tra sự tương quan theo phương pháp Pearson trước khi đưa vào các mô hình phân tích.

\section{KẾT QUẢ}

Có 74 bẫy chụp ảnh đã được đặt ngoài thực địa tại VQG LG-XM với tồng thời gian là 4.818 đềm, trong đó có 56 máy có ghi nh ận hình ảnh $L$. diardi (hình 2) và 28 máy ghi nhận có xuất hiện con người hoạt động trong khu vực nghiên cứu (hình 3 ).

Kết quả phân tích sự hiện diện của $L$. diardi cho thấy, xác suất bắt gặp trung bình của chúng lần lượt là $0,84(0,69-0,92, \mathrm{SE}=$ $0,06)$ và $0,19(0,16-0,23 ; \mathrm{SE}=0,02)$ trong điều kiện không bị chi phối bởi các yếu tố môi trường. 


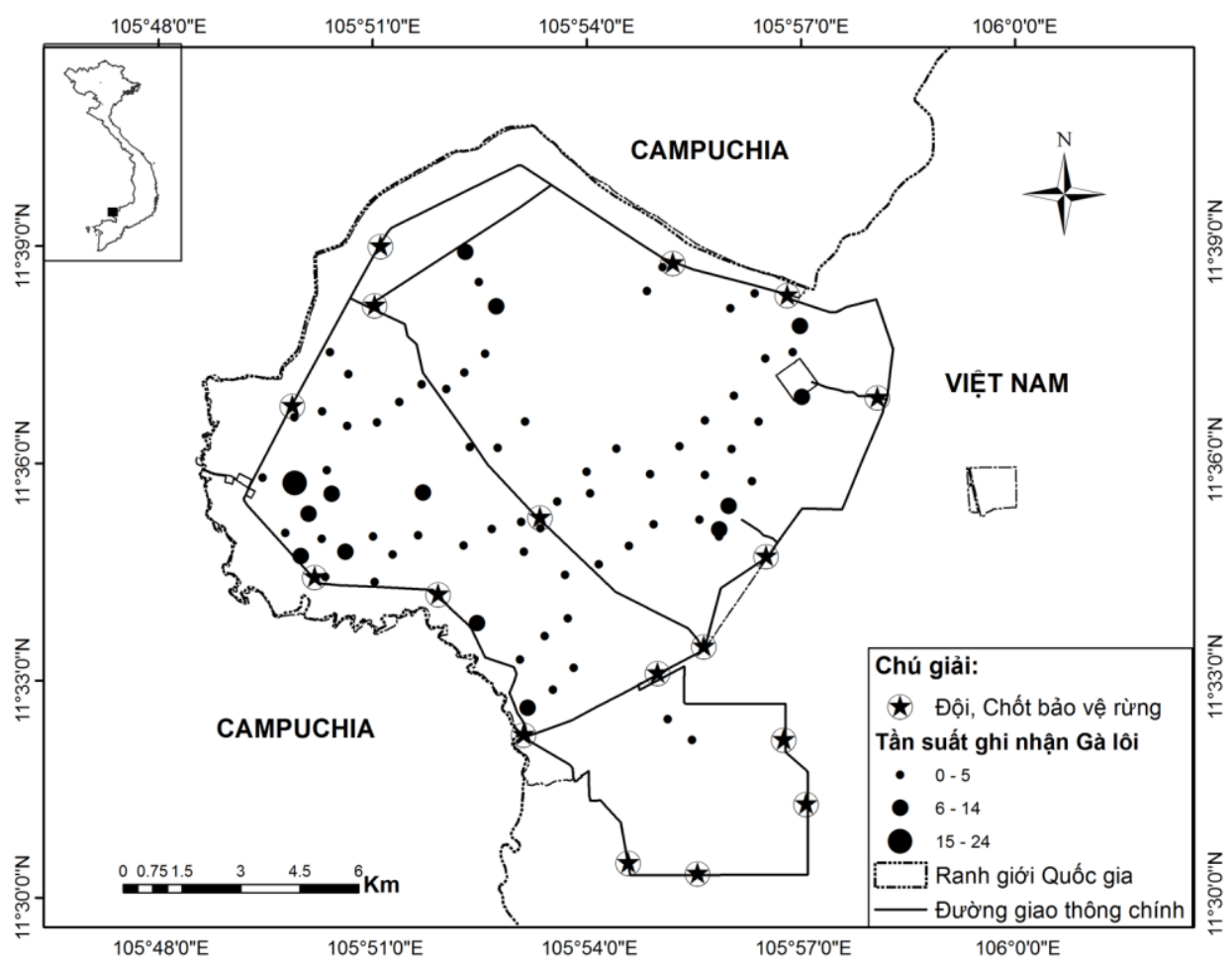

Hình 2. Tần suất hiện diện của gà lôi hông tía tại các bẫy chụp ảnh

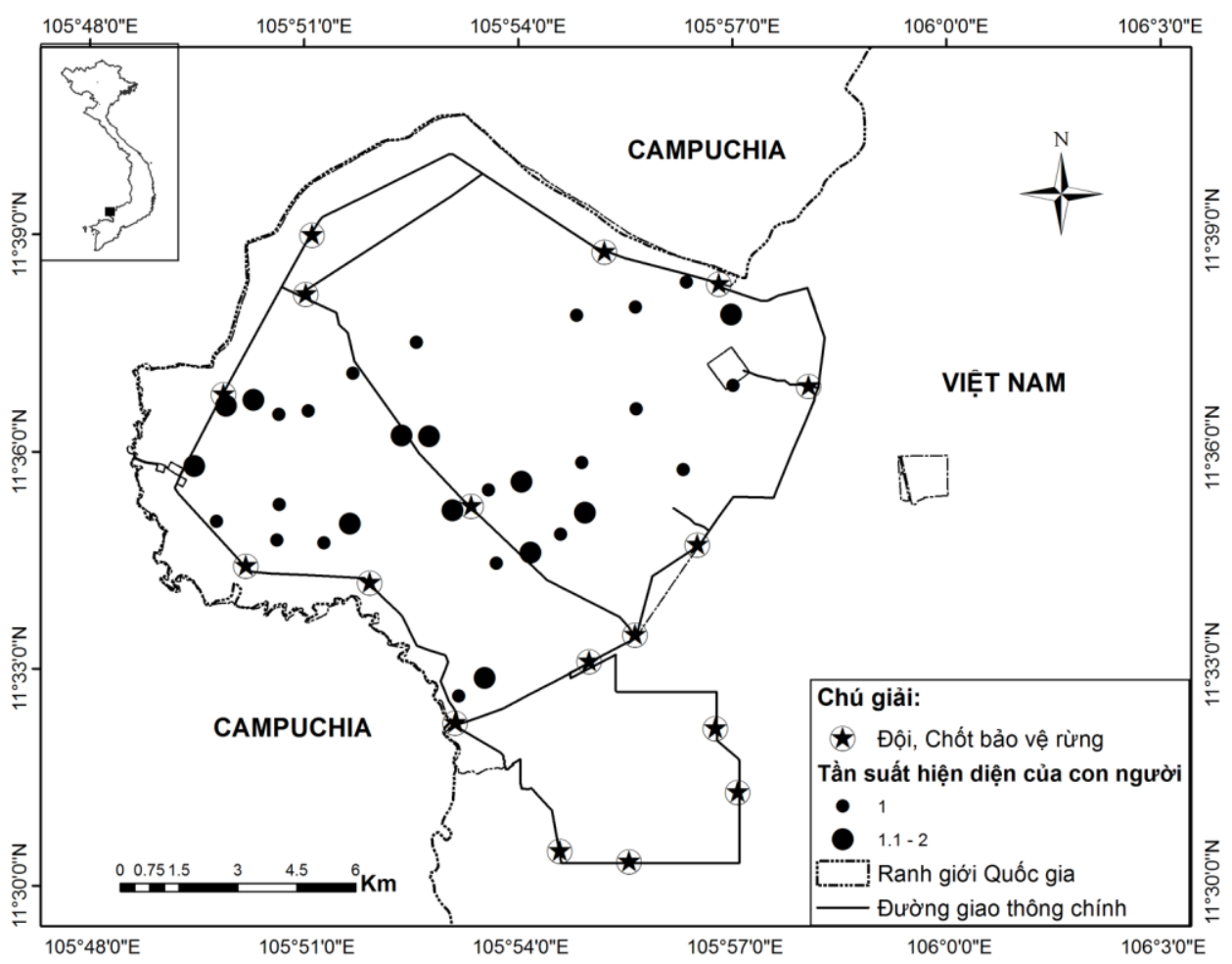

Hình 3. Tần suất hiện diện của con người trong khu vực nghiên cứu 
Các yếu tố ảnh hưởng đến độ phong phú của $L$. diardi

Có 16 mô hình tương quan phân phối Poisson đã được xây dựng kể cả mô hình không biến số. Mô hình tốt nhất bao gồm ba yếu tố môi trường đó là độ che phủ của rừng, khoảng cách đến các trạm bảo vệ rừng và tần suất hoạt động của con người trong khu vực nghiên cứu (bảng 1). Kết quả phân tích hệ số biến thiên của các yếu tố môi trường từ mô hình tốt nhất cho thấy $L$. diardi có xu hướng phân bố trong sinh cảnh rừng có độ che phủ cao $(\beta=0,41)$ ở những khu vực gần các trạm bảo vệ rừng $(\beta=-0,25)$ và khu vực ít có sự hiện diện của người dân $(\beta=-0,22)$ (bảng 2 ).

Bảng 1. Hai mô hình tốt nhất về tương quan giữa các yếu tố môi trường với số lượng của gà lôi hông tía (L. diardi) được lựa chọn từ 16 mô hình đã xây dựng. Mô hình có 3 biến số bao gồm độ che phủ của rừng, khoảng cách đến các trạm bảo vệ rừng và tần suất hiện diện của con người có chỉ số $\mathrm{AICc}$ thấp nhất $(\mathrm{AICc}=1110,28)$ và mức độ thích hợp với thực tế cao nhất $(\mathrm{AICcWt}=0,60)$

\begin{tabular}{|c|c|c|c|c|c|}
\hline Các yếu tố ảnh hường & Tham số & $\mathrm{AICc}$ & Delta AICc & $\mathrm{AICcWt}$ & Cum.Wt \\
\hline $\begin{array}{l}\text { 1. Độ che phủ của rừng, } \\
\text { 2. Khoảng cách đến trạm bảo vệ rừng } \\
\text { 3. Tần suất hiện diện của con người }\end{array}$ & 4 & $1.110,28$ & 0 & 0,60 & 0,60 \\
\hline $\begin{array}{l}\text { 1. Độ che phủ của rừng, } \\
\text { 2. Khoảng cách đến trạm bảo vệ rừng } \\
\text { 3. Tần suất hiện diện của con người } \\
\text { 4. Độ dày của thảm thực vật }\end{array}$ & 5 & $1.111,42$ & 1,14 & 0,34 & 0,94 \\
\hline
\end{tabular}

Ghi chú: $\mathrm{K}$ là số lượng tham số trong mô hình, $\triangle \mathrm{AICc}$ là sự khác nhau giữa $\mathrm{AICc}$ của các mô hình đã phân tích; Mô hình có giá trị $\Delta \mathrm{AICc}=0$ là mô hình có khả năng được chọn cao nhất. Mô hình có giá trị $\triangle \mathrm{AICc}$ trong khoảng $0-2$ thì khả năng được chọn ở mức trung bình, giá trị này lớn hơn 2 thì khả năng được lựa chọn rất thấp. Wi: mức độ thích hợp của mô hình so với thực tế.

Bảng 2. Hệ số biến thiên của các yếu tố môi trường trong mô hình tốt nhất

\begin{tabular}{|l|c|c|c|c|}
\hline \multicolumn{1}{|c|}{ Các yếu tố ảnh hưởng } & $\begin{array}{c}\text { Hệ số } \\
\text { biến thiên }\end{array}$ & $\begin{array}{c}\text { Sai số } \\
\text { chuấn }\end{array}$ & $\begin{array}{c}\text { Ngưỡng } \\
\text { dưới } 95 \%\end{array}$ & $\begin{array}{c}\text { Ngưỡng trên } \\
95 \%\end{array}$ \\
\hline Hệ số chặn & $-1,45$ & 0,08 & $-1,61$ & $-1,30$ \\
\hline Độ che phủ của rừng & 0,41 & 0,13 & 0,12 & 0,68 \\
\hline Khoảng cách đến trạm bảo vệ rừng & $-0,25$ & 0,07 & $-0,33$ & $-0,12$ \\
\hline Tần suất hiện diện của con người & $-0,22$ & 0,08 & $-0,39$ & $-0,07$ \\
\hline
\end{tabular}

\section{THẢO LUẬN}

Xác suất hiện diện trung bình của $L$. diardi trong khu vực nghiên cứu là 0,84 trong sinh cảnh rừng thường xanh có độ che phủ của rừng cao $(\beta=0.41)$. Xu hướng chọn nơi sinh sống có độ che phủ của rừng cao đã được ghi nhận ở các loài thuộc bộ Gà trong đó có $L$. diardi (Sukumal et al., 2010; Suwanrat et al., 2014), các loài khác như gà lôi Hume (Syrmaticus humiae) (Iamsiri \& Gale, 2008), các loài Gà so như Arborophila torqueola (Liao et al., 2007), Gà so Sichuan (Arborophila rufipectus) (Bo et al., 2009; Liao et al., 2008), Gà tiền mặt đỏ (Polyplectron germaini), Gà so cổ hung (Arborophila davidi), và Gà so ngực gụ (Tropicoperdix chloropus) (Vy et al., 2017a; Vy et al., 2017b). Sự lựa chọn kiểu sinh cảnh có đặc điểm như vậy có thể giúp chúng giảm nhẹ khả năng bị phát hiện bởi các loài ăn thịt, đặc biệt là các loài săn mồi từ trên cao.

Loài $L$. diardi hoạt động khá rộng trong khu vực nghiên cứu nhưng xác suất bắt gặp khá thấp ở mức 0,19 . Trước đây, chưa có nghiên cứu nào về sinh cảnh hay hiện trạng của $L$. diardi ở VQG-LGXM nên không thể đánh giá sự biến động về hiện trạng phân bố và xác suất bắt gặp loài này tại VQG-LGXM 
theo thời gian. Tuy nhiên, với sinh cảnh sống phù hợp (độ che phủ trung bình của rừn $\mathrm{g}$ cao $0,93 \%, \mathrm{SE}=0,006$ ), độ dày trung bình của thảm thực vật cao và khá tương đồng trong toàn khu vực nghiên cứu $(0,75 \%, \mathrm{SE}=0,011)$, khả năng sinh sản của $L$. diardi cao $(6,4 \pm 0,3$ trứng/lứa) (Suwanrat et al., 2014) thì xác suất bắt gặp ở mức 0,19 được xem là rất thấp so với điều kiện môi trường sinh thái ở đây. Ngoài các yếu tố môi trường tự nhiên, sự hiện diện của $L$. diardi chắc chắn chịu ảnh hưởng của những yếu tố có liên quan đến hoạt động của con người.

Kết quả nghiên cứu cho thấy, độ phong phú của $L$. diardi có tương quan nghịch với khoảng cách đến các trạm bảo vệ̣ rừng $(\beta=0,25)$ và sự xuất hiện của con người $(\beta=0,22)$. Rõ ràng, độ phong phú của loài giảm dần ở những khu vực xa trạm bảo vệ rừng và những nơi có sự xuất hiện của con người thường xuyên hơn do không bị kiểm soát. Vị trí và hoạt động tuần tra của các trạm như hiện nay có thể ngăn chặn các vụ xâm nhập trái phép ngay từ ngoài Vườn quốc gia. Dũ liệu thu được từ bẫy chụp ảnh cho thấy hầu hết những khu vực có tần suất xuất hiện cao của gà lôi hông tía (hình 2) và con người ít xuất hiện (hình 3) đều là những khu vực gần trạm bảo vệ rừng và kết quả ngược lại ở những khu vực xa trạm.

Kết quả nghiên cứu này cũng cho thấy các hoạt động tuần tra ở những khu vực xa trạm bảo vệ rừng cần tăng cường hơn so với những khu vực gần trạm. Thực tế cho thấy, các trạm bảo vệ rừng đóng vai trò rất quan trọng trong việc thực thi pháp luật, ngăn chặn các hoạt động săn bắt trong các khu bảo tồn (Ghoddousi et al., 2016; Jenks et al., 2012) cũng như góp phần định hình sự phân bố của nhiều loài động vật theo không gian (Dajun et al., 2006; Hunter \& Cresswell, 2015; Jenks et al., 2012). Những khu vực xa trạm bảo vệ rừng thường có các hoạt động săn bắt cao hơn và mật độ của nhiều loài động vật thấp hơn so với các khu vực gần trạm (Ghoddousi et al., 2016; Hunter \& Cresswell, 2015; Jenks et al., 2012). Việc tăng cường giám sát, đánh giá tần suất và qui mô của các hoạt động tuần tra bảo vệ rừng hàng năm góp phẩn nâng cao hiệu quả của công tác bảo tồn vì đã làm giảm rất nhiều số vụ săn bắt trong các khu bảo tồn tại châu Phi (Hilborn et al., 2006; Jachmann, 2008). Vì vậy, ở VQG LG-XM, để nâng cao hiệu quả của các hoạt động tuần tra bảo vệ rừng cần đẩy mạnh công tác quản lý và tăng cường giám sát hoạt động ở khu vực xa các trạm bảo vệ rừng.

\section{Mối đe dọa đến Lophura diardi}

Kết quả phân tích cho thấy tần suất hiện diện của con người (người dân) có tương quan nghịch với sự hiện diện và số lượng của gà lôi hông tía (hình 2,3 và bảng 2 ). Theo báo cáo của VQG-LGXM, trung bình mỗi năm số lượng bẫy dây cáp (bẫy giò) do nhân viên của Vườn tháo gỡ khoảng 1.750 chiếc cùng với các dụng cụ săn bắt khác như lưới và súng săn. Ngoài ra, qua những dữ liệu từ bẫy ảnh cho thây con người xuất hiện trong rừng cùng với súng săn, chó săn và các lâm sản ngoài gỗ (tổng số hình ảnh về người dân vào rừng từ bẫy chụp ảnh là 42), chúng tôi cho rằng sự hiện diện của họ trong rừng có những tác động đáng kể đến tài nguyên và môi trường sống ở đây trong đó có hoạt động săn bắt.

\section{KÊT LUẬN}

Sự thay đổi của sinh cảnh sống (habitat) có ảnh hưởng đến phân bố và hoạt động của Gà lôi hông tía, Lophura diardii. Vì vậy, điều này cần được chú ý trong hoạt động quản lý và bảo tồn loài này tại VQG LG-XM. Kết quả nghiên cứu này cho thấy, Gà lôi hông tía phân bố rộng trong sinh cảnh rừng thường xanh nhưng xác suất bắt gặp thấp mà ngu yên $n h a ̂ n$ chính do việc tuần tra ở các khu vực xa trạm bảo vệ rừng chưa thường xuyên, và đây cũng là hạn chế lớn trong nỗ lực bảo tồn loài này ở VQG.

\section{TÀI LIỆU THAM KHẢO}

Abrams J. F., Axtner J., Bhagwat T., Mohamed A., Nguyen A., Niedballa J., Sollmann R., Tilker A., Wilting A., 2018. Studying terrestrial mammals in tropical rainforests. A user's guide for cameratrapping and environmental DNA. Leibniz-IZW, Berlin, Germany, pp. 88. 
Nguyen Tran Vyetal.

Akaike H. 1973. Information theory and an extension of the maximum likelihood principle. In B. N. Petrovand \& F. Csàki (Eds.), 2nd International Symposium on Information Theory, Akadémiai Kiàdo,Budapest, Hungary pp. 267-281.

Anonymous, 2007a. Inverstigating status of fauna and flora in Lo Go - Xa Mat National park. Viện Sinh học Nhiệt đới.

Anonymous, 2007b. Vietnam Red Data Book (Part 1 - Animals). Publishing House of Natural Sciences and Technology, Ha Noi, pp. 515 .

BirdLife International, 2019. Lophura diardi. The IUCN Red List of Threatened Species 2016: e.T22679274A92808547. http://dx.doi.org/10.2305/IUCN.UK.20163.RLTS.T22679274A92808547.en. [accessed 24 July, 2019].

Bo D., Dowell S. D., Garson P. J., Fen-Qi, H., 2009. Habitat utilisation by the threaten ed Sichuan partridge Arborophila rufipectus: consequences for managing newly protected areas in southern China. Bird Conserv. Int, 19(2): 187-198.

Brickle N. W., Duckworth J., Tordoff A. W., Poole, C. M., Timmins, R., McGowan, P. J., 2008. The status and conservation of Galliformes in Cambodia, Laos and Vietnam. Biodiversity and Conservation, 17(6): 1393-1427.

Burnham K. P., Anderson D. R., 2002. Model selection and multimodel inference: a practical information-theoretic approach $\left(2^{\text {nd }}\right.$ ed.). Springer Science \& Business Media, New York, pp. 482.

Dajun W., Sheng L., McShea W. J., Fu L. M., 2006. Use of remote-trip cameras for wildlife surveys and evaluating the effectiveness of conservation activities at a nature reserve in Sichuan Province, China. Environmental Management, 38(6): 942-951.

Duckworth J., Batters G., Belant J., Bennett E., Brunner, J., Burton, J., Challender, D., Cowling, V., Duplaix, N., Harris, J., 2012.
Why South-East Asia should be the world's priority for averting imminent species extinctions, and a call to join a developing cross-institutional programme to tackle this urgent issue. J S.A.P.I.EN.S, 5(2): 1-19.

Fiske I., Chandler R., Miller D., Royle A., Kery, M., Hostetler, J., Hutchinson, R., Smith, A., Kellner, K., Royle, M. A., 2019. Package 'unmarked'.

Gelman A., 2008. Scaling regression inputs by dividing by two standard deviations. Stat Med, 27(15): 2865-2873.

Ghoddousi A., Kh. Hamidi A., Soofi M., Khorozyan I., Kiabi B., Waltert M., 2016. Effects of ranger stations on predator and prey distribution and abundance in an I ranian steppe landscape. Animal Conservation, 19(3): 273-280.

Grainger M. J., Garson P. J., Browne S. J., McGowan P. J., Savini, T., 2018. Conservation status of Phasianidae in Southeast Asia. Biological Conservation, 220: 60-66.

Grainger M. J., Ngoprasert D., McGowan P. J., Savini, T., 2017. Informing decisions on an extremely data poor species facing imminent extinction. Oryx, 51(4): 1-7.

Gray T. N., Hughes A. C., Laurance W. F., Long, B., Lynam A. J., O'Kelly H., Ripple, W. J., Seng, T., Scotson, L., Wilkinson, N. M., 2018. The wildlife snaring crisis: an insidious and pervasive threat to biodiversity in Southeast Asia. Biodiversity and Conservation, 27(4): 1031-1037.

Hilborn R., Arcese P., Borner M., Hando J., Hopcraft G., Loibooki M., Mduma S., Sinclair, A. R., 2006. Effective enforcement in a conservation area. Science, 314(5803): 1266-1266.

Hilton-Taylor C., Pollock C. M., Chanson J. S., Butchart S. H., Oldfield T. E., Katariya, V., 2009. State of the world's species (J.-C. Vié, C. Hilton-Taylor \& S. N. Stuart Eds.). IUCN, Gland, Switzerland, pp. 184 
Hossain A. N. M., Barlow A., Barlow C. G., Lynam, A. J., Chakma, S., Savini, T., 2016. Assessing the efficacy of camera trapping as a tool for increasing detection rates of wildlife crime in tropical protected areas. Biological Conservation, 201: 314-319.

Hunter M., Cresswell W., 2015. Factors affecting the distribution and abundance of the Endangered volcano rabbit Romerolagus diazi on the Iztaccihuatl volcano, Mexico. Oryx, 49(2): 366-375.

Iamsiri A., Gale G. A., 2008. Breeding season habitat use by Hume's pheasant Syrmaticus humiae in the Doi Chiang Dao Wildlife Sanctuary, Northern Thailand. Zool Stud, 47(2): 138-145.

IUCN 2019. Threatened species in each country (totals by taxonomic group). https://www.iucnredlist.org/resources/sum mary-statistics\#Summary\%20Tables. [accessed 22 July, 2019].

Jachmann H., 2008. Illegal wildlife use and protected area management in Ghana. Biological Conservation, 141(7): 1906-1918.

Jenks K. E., Howard J., Leimgruber P., 2012. Do ranger stations deter poaching activity in national parks in Thailand? Biotropica, 44(6): 826-833.

Liao W. B., Fuller R. A., Hu J. C., Li C., 2008. Habitat use by endangered Sichuan partridges Arborophila rufipectus during the breeding season. Acta Ornithol, 43(2): 179-184.

Liao W. B., Hu J. C., Li C., 2007. Habitat utilization during the pairing season by the common hill partridge Arborophila torqueola in Baiposhan Natural Reserve, Sichuan, China. Ornithol Sci, 6(2): 87-94.

MacKenzie D. I., 2006. Occupancy estimation and modeling: inferring patterns and dynamics of species occurrence. Academic Press, pp. 344

McGowan P. J., Garson, P. J., 2002. The Galliformes are highly threatened: should we care? Oryx, 36(04): 311-312.
Mugerwa B., Sheil D., Ssekiranda P., Heist, M., Ezuma, P., 2013. A camera trap assessment of terrestrial vertebrates in Bwindi Impenetrable National Park, Uganda. African Journal of Ecology, 51(1): 21-31.

Niedballa, J., Courtiol, A., Sollmann, R. (2017) camtrapR: camera trap data management and preparation of occupancy and spatial capture-recapture analyses. R package version 0.99. 9 .

Development Core Team, 2019. R: A language and environment for statistical computing. http://www.R-project.org/. [accessed 5 January, 2019].

Rovero F., Tobler M., Sanderson J., 2010. Camera trapping for inventorying terrestrial vertebrates. Manual on field recording techniques and protocols for All Taxa Biodiversity Inventories and Monitoring. The Belgian National Focal Point to the Global Taxonomy Initiative: 100-128.

Sukumal N., Gale G. A., Savini T., 2010. Submontane habitat selection by a lowland pheasant. Raffles Bull. Zool, 58(2): 391-401.

Sukumal N., McGowan P. J., Savini T., 2015. Change in status of green peafowl Pavo muticus (Family Phasianidae) in Southcentral Vietnam: A comparison over 15 years. Glob Ecol Conserv, 3(1): 11-19.

Suwanrat J., Ngoprasert D., Sukumal N., Suwanwaree P., Savini T., 2014. Reproductive ecology and nest-site selection of Siamese fireback in lowland forest. Raffles Bull. Zool, 62(1): 581-590.

Tilker A., Nguyen A., Abrams J. F., Bhagwat, T., Le, M., Van Nguyen, T., Nguyen, A. T., Niedballa, J., Sollmann, R., Wilting, A., 2018. A little-known endemic caught in the South-east Asian extinction crisis: The Annamite striped rabbit Nesolagus timminsi. Oryx: $1-10$.

Vy N. T., Ngoprasert D., Browne S., Savini T., 2017a. Status and range decline of two 
galliformes species in Southeast Asia. Bird Conserv. Int, 27(4): 1-16. doi: 10.1017/S0959270917000168

Vy N. T., Ngoprasert D., Gale G. A., Browne, S. J., Savini, T., 2017b. Co-occurrence of two sympatric galliform species on a landscape-scale. Raffles Bulletin of Zoology, 65(1): 60-67.
Wege D. C., Long A. J., Vinh M. K., Dung V. V., Eames J. C., 1999. Expanding the protected areas network in Vietnam for the 21st century: an analysis of the current system with recommendations for equitable expansion. BirdLife International Vietnam Programme, $\mathrm{Ha}$ Noi, Vietnam, pp. 74. 